\title{
Three-dimensional reconstruction of myocardial contrast perfusion from biplane cineangiograms by means of linear programming techniques
}

\author{
Adrie C.M. Dumay, ${ }^{*}$ H. Minderhoud, ${ }^{*}$ J.J. Gerbrands, F. Zijlstra, C.E. Essed, P.W. Serruys \& J.H.C. \\ Reiber \\ Thoraxcenter, Erasmus University, Rotterdam, the Netherlands; ${ }^{*}$ Department of Electric Engineering, Delft \\ University of Technology, Delft, the Netherlands
}

Accepted 9 December 1987

Key words: three-dimensional reconstruction, myocardial contrast perfusion, coronary flow reserve, biplane cineangiography, quantitative coronary angiography, quantitative left ventriculography, linear programming techniques

\section{Summary}

The assessment of coronary flow reserve from the instantaneous distribution of the contrast agent within the coronary vessels and myocardial muscle at the control state and at maximal flow has been limited by the superimposition of myocardial regions of interest in the two-dimensional images. To overcome these limitations, we are in the process of developing a three-dimensional (3D) reconstruction technique to compute the contrast distribution in cross sections of the myocardial muscle from two orthogonal cineangiograms. To limit the number of feasible solutions in the 3D-reconstruction space, the 3D-geometry of the endo- and epicardial boundaries of the myocardium must be determined. For the geometric reconstruction of the epicardium, the centerlines of the left coronary arterial tree are manually or automatically traced in the biplane views. Next, the bifurcations are detected automatically and matched in these two views, allowing a 3D-representation of the coronary tree. Finally, the circumference of the left ventricular myocardium in a selected cross section can be computed from the intersection points of this cross section with the 3D coronary tree using B-splines. For the geometric reconstruction of the left ventricular cavity, we envision to apply the elliptical approximation technique using the LV boundaries defined in the two orthogonal views, or by applying more complex 3D-reconstruction techniques including densitometry. The actual 3D-reconstruction of the contrast distribution in the myocardium is based on a linear programming technique (Transportation model) using cost coefficient matrices. Such a cost coefficient matrix must contain a maximum amount of a priori information, provided by a computer generated model and updated with actual data from the angiographic views. We have only begun to solve this complex problem. However, based on our first experimental results we expect that the linear programming approach with advanced cost coefficient matrices and computed model will lead to acceptable solutions in the 3D-reconstruction of the myocardial contrast distribution from biplane cineangiograms. 


\section{Introduction}

The severity of a coronary obstruction can be described in terms of its geometry (morphologic severity), as well as in terms of its effect on the perfusion of the underlying myocardium (functional or physiologic severity). Although the morphologic severity of coronary obstructions can be measured with high accuracy and precision with computer-assisted image processing techniques [1], the physiologic or functional significance of an obstruction in an individual patient cannot be determined with sufficient accuracy from the geometry of the narrowing alone; there are many factors which may influence the mathematical formulas relating the geometric and functional descriptions, such as collateral circulation, previous myocardial infarction, etc. Despite these limitations, Gould et al. and Zijlstra et al. have attempted with increasing success to determine the coronary flow reserve from the pressure gradient-flow relation, which is based on the geometric description of a coronary obstruction $[2,3]$. Until such approaches have proven to be reliable predictors of coronary flow reserve in an individual patient, other techniques should be used to assess the functional consequences of coronary obstructions.

New developments in X-ray imaging introduced by Vogel et al. have led to the computation of relative regional myocardial flow in the control and maximal flow states by means of contrast medium appearance techniques following the administration of a coronary vasodilator [4]. By means of this approach, coronary flow reserve (CFR), defined as the ratio of maximum coronary flow after a maximal vasodilatory stimulus to resting flow, can be determined. With the introduction of intracoronary papaverine, maximal coronary blood flow can be safely induced during routine cardiac catherization $[5,6]$. Although this technique is usually applied on-line to video images of the coronary arterial system acquired directly from the image intensifier of the X-ray system, we have implemented this technique for off-line analysis from cinefilm $[3,7]$.

However, the present CFR imaging technique is limited by the fact that two-dimensional images of a three-dimensional structure are analyzed. In these two-dimensional images there does not exist a oneto-one relation between a selected myocardial region of interest (ROI) and one particular coronary segment perfusing that area due to overprojection of myocardial regions in front of and behind the selected region of interest perfused by other arterial segments. For some time, our group as well as Onnasch et al. have been studying the possibilities of three-dimensional reconstruction of the myocardial perfusion from two orthogonal projections to overcome these problems of superimposition [8, 9]. Our approaches to this complex problem will be discussed in this paper.

\section{Three dimensional reconstruction by means of linear programming techniques}

The linear programming (LP) approach for the 3D-reconstruction of the left ventricular lumen from biplane cineangiograms was introduced by Slump et al. [10]. Reiber et al. described the application of LP-techniques to the 3D-reconstruction of coronary arterial segments [11]. This technique could be improved by modelling the X-ray images of the contrast agent filled coronary vessel with a Poisson process [12]. Both applications were performed with the linear programming transportation model [13]. In this model a priori knowledge about the geometry of the left ventricular or coronary cross section was contained in the so-called cost coefficient matrix. Dumay has demonstrated that this LP-technique is also applicable to the 3Dreconstruction of myocardial perfusion from two projections; however, the definition of the appropriate cost coefficient matrix is crucial to the quality of the final results [8].

For the 3D-reconstruction of the myocardial perfusion from two orthogonal projections five major steps are proposed: 1 ) determination of the optimal biplane angiographic views; 2 ) myocardial perfusion image acquisition; 3) preprocessing of the selected myocardial perfusion images; 4) estimation of the 3D-geometry of the endo- and epicardial boundaries of the left ventricular myocardium and; 5) reconstruction of the regional myocardial perfu- 
sion in the different cross sections perpendicular to the left ventricular long axis. These major steps will be described in more detail in the following sections. The first experimental results are presented and discussed in section 8 .

\section{Determination of the optimal biplane angio- graphic views}

To optimize the limited amount of information available, the biplane angiographic views for the registration of the myocardial perfusion should be taken parallel to the direction of the left ventricular (LV) long axis. This means that at the start of the angiographic procedure, a routine biplane left ventricular angiogram must be acquired for the definition of the LV long axis. Immediately thereafter, end-diastolic biplane images of the contrast-filled left ventricle are replayed on the video monitors of the catheterization room and the cardiologist or a technician defines the LV long axis direction in the two views with the help of a personal computer with image digitizing capability and a mouse or joystick for user-interaction. The LV long axis in a 2D-image is defined as the line connecting the apex with the center of the short axis connecting the two aortic valve positions at the base of the left ventricle. From these data the $3 \mathrm{D}$-direction of the $\mathrm{LV}$ long axis can be computed. If the geometry of the $\mathrm{X}$-ray system is also available to the $\mathrm{PC}$, the optimal angiographic views for this study can be determined and the X-ray system positioned in these optimal settings for the remaining coronary and left ventricular angiograms [14].

\section{Myocardial perfusion image acquisition}

For the biplane image acquisition, the same protocol is followed as has been used for our monoplane studies $[3,7]$. In these studies, the heart was atrially paced at a rate just above the spontaneous heart rhythm. The nonionic contrast agent Iopamidol ${ }^{\circledR}$ was injected at $37^{\circ} \mathrm{C}$ into the coronary arterial system with an ECG-triggered Medrad Mark IV infusion pump. For the left coronary artery 7 or
$8 \mathrm{ml}$ was injected at a flow rate of 4 or $5 \mathrm{ml} / \mathrm{sec}$. For the right coronary artery $5 \mathrm{ml}$ was injected at a flow rate of $3 \mathrm{ml} / \mathrm{sec}$. The injection rate of the contrast medium is judged to be adequate when backflow of contrast medium into the aorta occurs. The angiogram is repeated $30 \mathrm{sec}$ after pharmacologically induced hyperemia by a bolus injection of $12.5 \mathrm{mg}$ papaverine into the coronary artery. The film speed for the biplane coronary cineangiograms is taken at 50 frames/sec.

\section{Digitization and preprocessing of the selected images}

For the quantitation of the relative coronary blood flow, five to six end-diastolic (ED)-cineframes in each of the two (biplane) image series are selected from successive cardiac cycles. These cineframes are digitized with a high-quality cinedigitizer at a resolution of $512 \times 512$ pixels and 256 grey levels $[3,14,15]$. The digitized images are corrected for the dark current of the video camera in the cinedigitizer. Logarithmic maskmode background subtraction is applied to the image subset to eliminate non-contrast medium densities. The last ED-cineframe prior to the contrast administration is chosen as the mask. The intensity level in the background subtracted cineangiograms has been found to be proportional to the irradiated amount of contrast material [14]. An example of a series of 6 consecutive background subtracted ED-cineframes of a right coronary artery in the RAO-projection is shown in Fig. 1. A corresponding sequence of images can be obtained from the orthogonal view.

The 3D-reconstruction of the myocardial perfusion must be determined for each pair of end-diastolic cineframes. In these reconstructed cross sections, the relative regional blood flow can then be calculated within user-defined myocardial regionsof-interest (ROI's) on the basis of the relative regional vascular volume and the mean contrast appearance time, as has been described in detail for the monoplane studies $[3,7,15]$.

As explained above, a 3D-reconstruction of myocardial contrast agent perfusion must be carried out for each end-diastolic moment. In these 


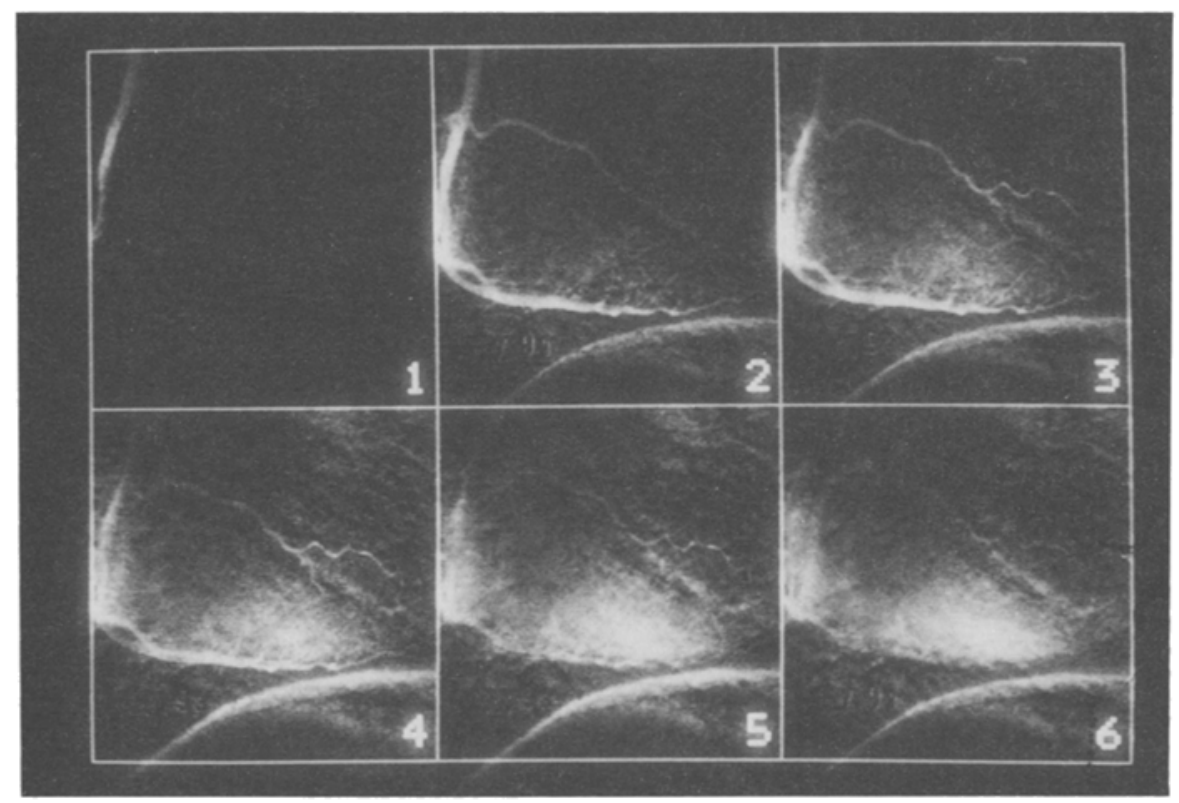

Fig. 1. End-diastolic cineframes, digitized in $512 \times 512$ eight-bit matrices from 6 consecutive heart beats following contrast injection. Stationary background structures were eliminated by means of logarithmic mask-mode background subtraction using the ED-cineframe acquired prior to the contrast administration as a mask.

reconstructions, a priori information is essential to limit the large number of possible solutions. We propose to use the geometry of the myocardial muscle to obtain a bounded volume in which the reconstruction must take place. How to obtain such geometric information will be discussed in the following section.

\section{Reconstruction of geometry}

The three-dimensional reconstruction of the myocardial perfusion in cross sections of the myocardium from only two orthogonal views is an underdetermined problem. Without additional a priori information, many solutions will fit the projection data. This makes clear that as much a priori information as possible must be incorporated into the reconstruction process to limit the collection of permissable solutions.

The size and shape of the left ventricular myocardial cross section are determined by the endocardial and epicardial boundaries. We envision that the endocardial boundaries be estimated from the left ventricular cineangiograms and the epicardial boundaries from the coronary arterial tree available from the coronary angiograms. That would mean that in addition to the angiographic investigation for the assessment of coronary flow reserve described above, a second biplane left ventriculography must be performed in the optimal views. In a right dominant system, there is only one branch from the right coronary tree, the Posterior Descending branch, that perfuses the myocardial muscle of the left ventricle. In a left dominant system, this branch comes from the circumflex artery. This means that in all cases information about the 3Dstructure of the left coronary arterial system only seems sufficient to estimate the size and shape of the epicardial boundaries of the cross sections of the left ventricular myocardial muscle.

If the acquisition procedure described under section 4 is followed for the left coronary system, then this same information can be used for the definition of the epicardial geometry of the left ventricular muscle. However, if only the perfusion of the right coronary system is being studied, an additional left coronary angiography must be performed. Our 
present approaches to solve these problems are described in the following paragraphs.

\subsection{Geometric reconstruction of the epicardium}

The major branches of the coronary arterial tree lie directly upon the epicardial surface of the myocardium and can thus be used to determine the outer boundaries of the myocardium. The vessels from the left coronary arterial system which can be used for these purposes are the Left Anterior Descending artery (LAD) with the diagonal branches, the Left Circumflex Artery $(\mathrm{Cx})$ with the Obtuse Marginal branch and the Postero Lateral branch, and the Posterior Descending branch, if the system is left dominant. The septal branches of the LAD cannot be used as these penetrate the septal wall. Therefore, the boundaries of the septal portion of the left ventricular myocardium must be estimated from the known positions of the epicardial vessels using an appropriate model.

Computation of the epicardial boundaries of the left ventricular myocardial cross sections will require the following steps:

a) manual or automated tracing of the centerlines of the left coronary arterial tree in biplane views.

b) three-dimensional matching of the two coronary trees.

c) determination of intersection points of selected cross sections of the heart with the coronary tree; these cross sections are taken perpendicular to the left ventricular long axis.

d) computation of the left ventricular epicardial boundary of each cross section based on the known intersection points with the coronary tree and on an appropriate geometric model.

These different steps will be described in some more detail in the following paragraphs

\subsubsection{Manual or automated tracing of the left coro-} nary arterial tree in biplane views

End-diastolic frames in the two orthogonal views are selected at the corresponding moments in the angiographic investigation. All the approximate centerlines of the major vessels and their branches as mentioned earlier, if visible in the images, are traced manually or tracked by an automated centerline tracing technique [16]. The spatial positions of the bifurcations and other crossing points (overlap of vessels) are determined by the computer program. An example of manually traced centerlines in two orthogonal views of a left coronary arterial system is given in Fig. 2 .

\subsubsection{Three-dimensional matching of the two views of the coronary tree}

To be able to describe the coronary tree in three dimensions, the projection data of the tree in the two views must be matched. For these purposes, graph matching techniques can be applied [17]. Each projected and traced tree is represented by a graph; the nodes in the graph correspond with bifurcations, trifurcations and crossings in the traced coronary tree. The arcs in the graph correspond with vessel segments. If there are loops in a graph (due to overlap of vessels), all the possible loopless trees derived from the graph must be created for the matching procedure. Therefore, such a graph with one or more loops is split into a set of loopless trees by a syntax which describes which nodes are connected to each other and by which arcs. An example of a simple graph with a loop in one of the two views is shown in Fig. 3.

By means of a set of transformations each tree in one set will be transformed into each of the possible trees in the other set. This set of transformations include: 1) removal of a node; 2) insertion of a node; and 3) shift of a node. Each of these transformations are associated with a certain cost. This means that the cost of transforming one tree into another can be calculated as the sum of all the individual costs associated with the necessary transformations. The two trees that can be transformed into each other at the lowest total cost are assigned as the corresponding trees by the matching algorithm. Now that the corresponding nodes and arcs in the two orthogonal views are known, the $3 \mathrm{D}$-coordinates of the traced coronary tree can be computed. 

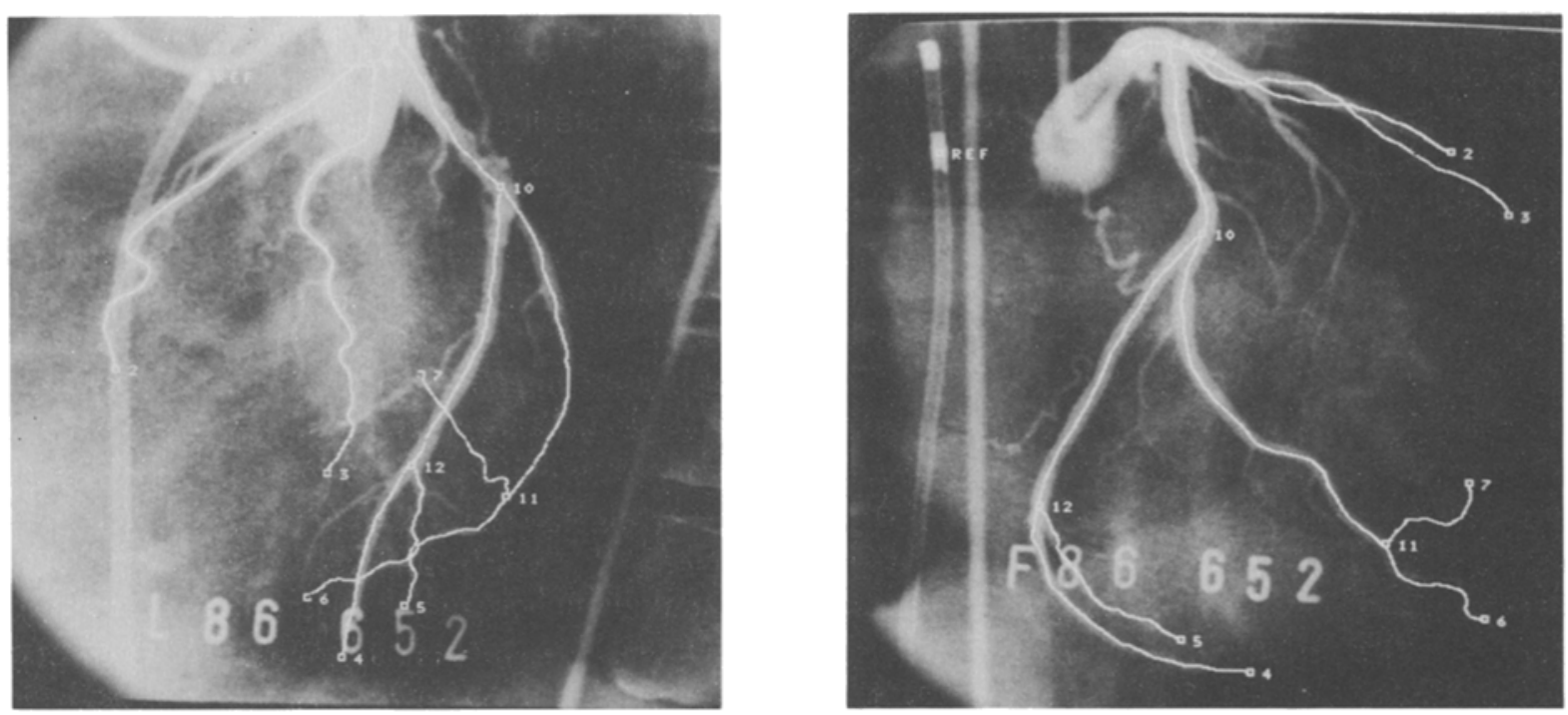

Fig. 2. Manually traced centerlines of left coronary arterial tree in biplane, orthogonal views (LAO at left, RAO at right).

\subsubsection{Hull fitting through the $3 D$ coronary tree} The three-dimensional coronary tree encompasses the myocardium. If we want to determine the distribution of the myocardial perfusion within cross sections of the left ventricular myocardial muscle, sliced perpendicular to the left ventricular long axis, the same cuts through the coronary tree structure must be made to obtain the information necessary for the definition of the outer boundaries of the myocardium. As the direction of the left ventricular long axis is known (see section 3), cross sections through the $3 \mathrm{D}$ tree structure perpendicular to the LV long axis can be defined and the corresponding intersection points with the major branches of the coronary arteries determined. On the basis of these intersection points the true size and shape of the left ventricular epicardium in such a cross section must be reconstructed. This is illustrated in Fig. 4. The epicardial boundary of the heart defines the circumference of the left ventricular myocardium, except at the septal region. For this portion of the muscle, an appropriate model must be used, such that it can be reconstructed from the known epicardial intersection points. Such a reconstruction certainly is not a trivial problem, since usually only 4 to 5 intersection points mainly situated along the frontal and lateral sides are available. An example of the tracings of the outer and inner boundaries of six cross sections through a postmortem heart with the coronary vessels indicated is shown in Fig. 5; we must be aware of the fact that the heart was fixed in the endsystolic phase.

For the reconstruction of the left ventricular epicardial boundary in a cross section on the basis of the known positions of the coronary vessels we have studied the following techniques: $2 \mathrm{D}$ ellips

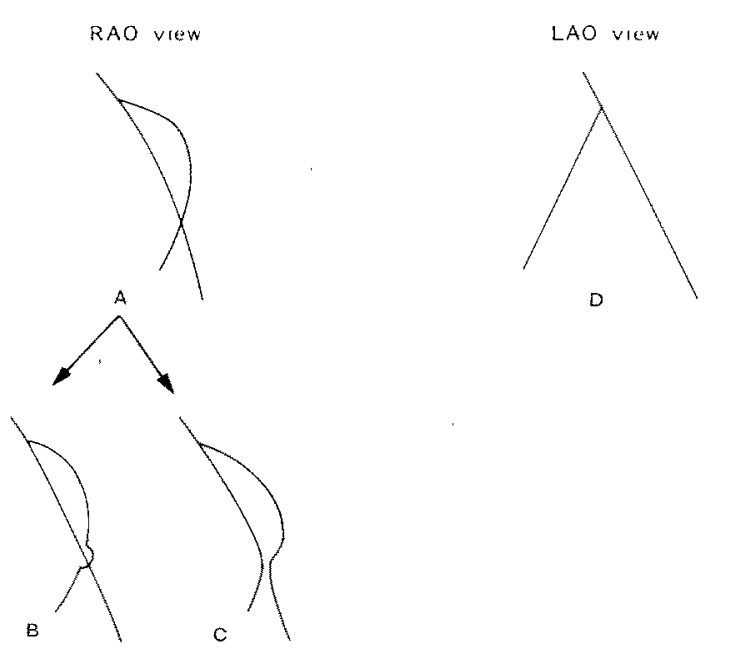

Fig. 3. The splitting of graph A (with loop) into a set of two trees $\mathrm{B}$ and $\mathrm{C}$ without loops; graph $\mathrm{D}$ represents the orthogonal view. 


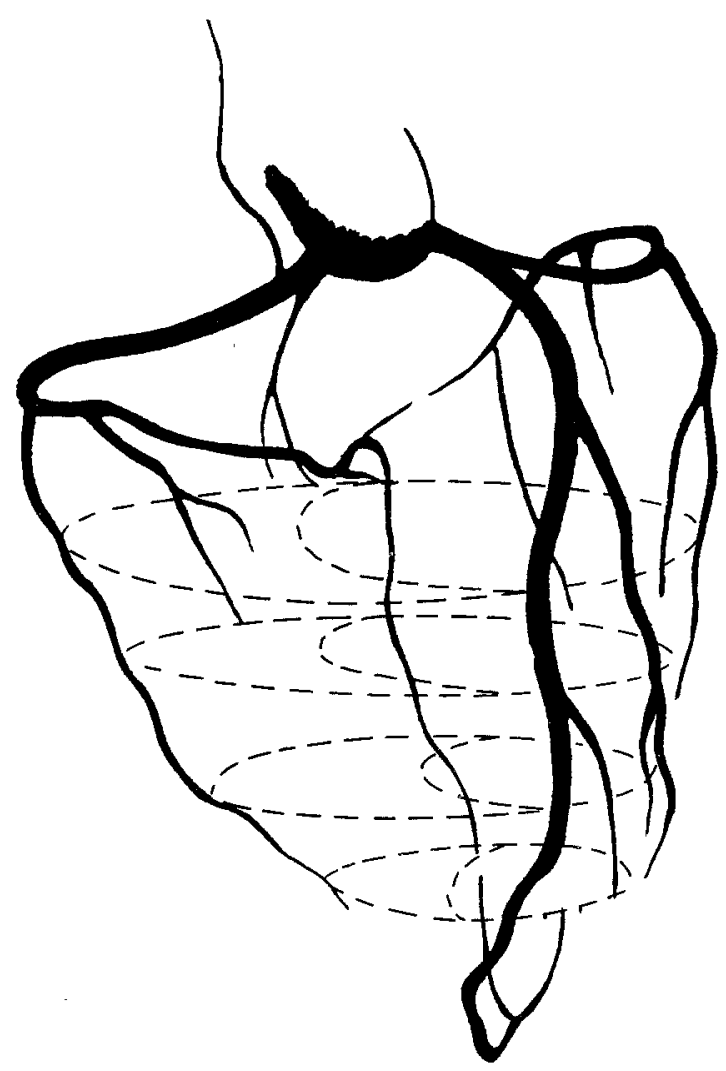

Fig. 4. Diagrammatic presentation of convex hulls describing the epicardial boundaries and the septal region of the left ventricular myocardium, and the epicardial boundaries of the entire myocardium.

and $3 \mathrm{D}$ ellipsoid model fitting, isotropic interpolation of polynomials and both 2D and 3D Spline fitting [18].

\subsection{Geometric reconstruction of the endocardium}

The a priori information of the epicardial boundaries in the cross sections is not sufficient. The size and shape of the endocardial boundaries of the myocardial muscle must be determined as well. That information must be obtained from the biplane left ventricular angiograms, that are available for a study of this type (introduction section $6)$.

We do have extensive experience with the automated contour detection of the left ventricle in the RAO-projection (Fig. 6) [19]. Similar techniques
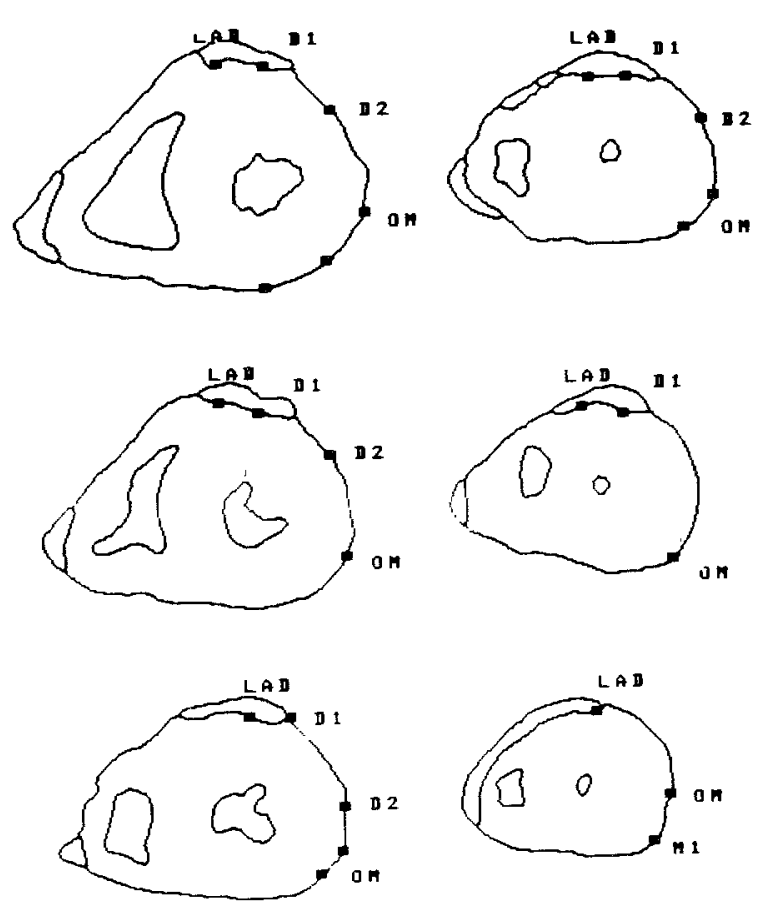

Fig. 5. Tracings of the outer boundaries of six cross sections through a postmortem heart with the intersections with the coronary vessels indicated.

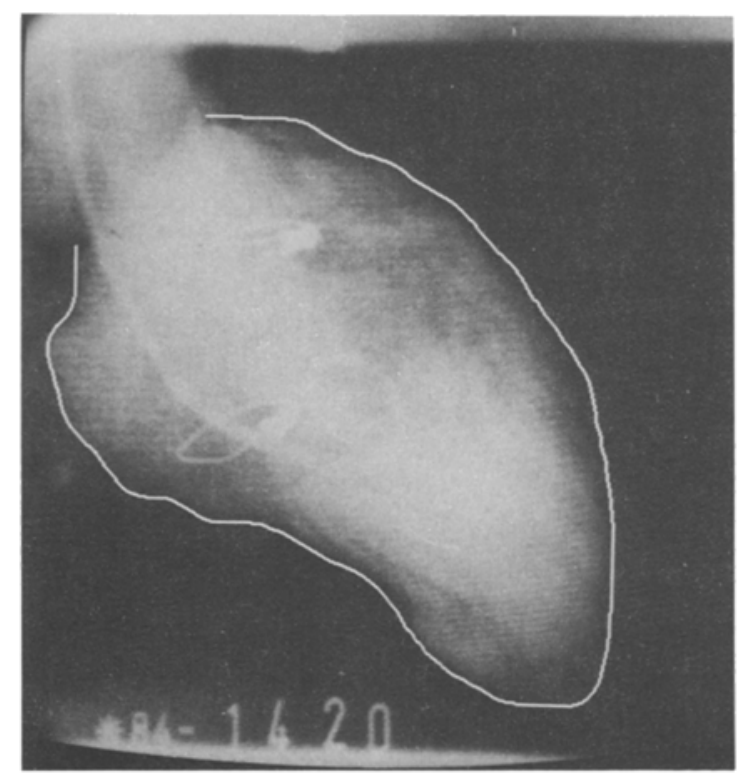

Fig. 6. Example of left ventricular cineangiogram in RAO-view with automatically detected boundaries. 
need to be developed for the LAO-angiographic projection. From the LV boundaries defined in the two orthogonal views, the size and shape of the various cross sections can be defined by elliptical approximation [20], being the simplest approach, or by applying more complex 3D-reconstruction techniques which also use the densitometric information in the LV-angiograms, as have been described by Slump et al. [10] and by Onnasch et al. [21]. Our first approach will be based on the relatively simple elliptical approximation.

\section{Reconstruction of regional myocardial perfusion}

Once the endo- and epicardial geometry of the left ventricular myocardium has been defined, the regional distribution of myocardial perfusion must be reconstructed from the preprocessed images. The 3D-reconstruction takes place on a slice-by-slice basis, as depicted in Fig. 7. As mentioned earlier, the reconstruction of the myocardial perfusion is a heavily underdetermined problem when only two projections are available. Suppose, the $2 \mathrm{D}$-projection images of the myocardial perfusion are defined in $\mathrm{M} \times \mathrm{M}$ and $\mathrm{N} \times \mathrm{N}$ matrices, respectively; in practice, $M=N$. Let $X$ denote an $M \times N$ matrix with entries $x(i, j) \varepsilon\{0,255\}$. The elements of $X$ are to be determined from the following set of equations:

$$
\begin{array}{ll}
\sum_{j=1}^{N} x(i, j)=\alpha(i) & i=1, \ldots, M \\
\sum_{i=1}^{M} x(i, j)=\beta(j) & j=1, \ldots, N
\end{array}
$$

where $\alpha(i), i=1, \ldots, M$ are the elements of the vector $\alpha$ containing the row sums of $X$ and $\beta(j)$ $j=1, \ldots, N$ the elements of the vector $\beta$ containing the column sums of $X$. This set of equations is not independent, since

$$
\sum_{i=1}^{M} \alpha(i)=\sum_{j=1}^{N} \beta(j)
$$

i.e. the total sum of the row projection data equals the total sum of the column projection data. Thus,

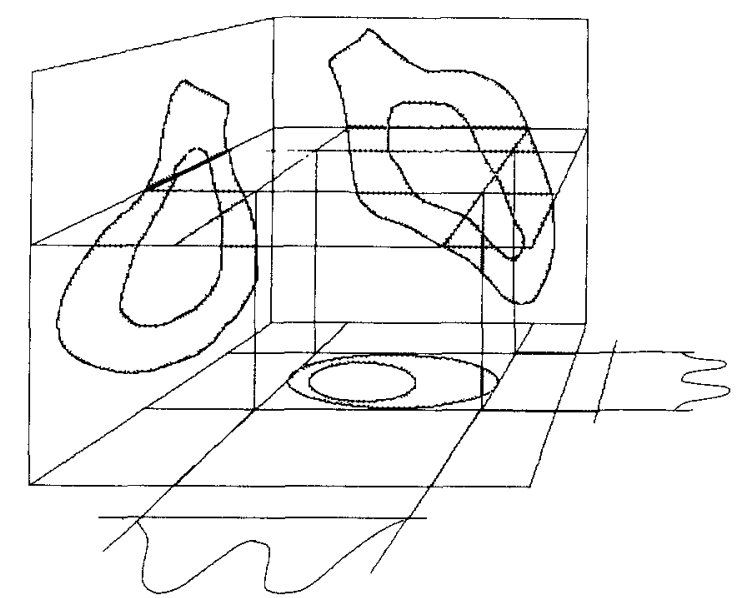

Fig. 7. Slice-wise 3D-reconstruction of myocardial perfusion in the volume enclosed by the epi- and endocardial boundaries.

$\mathrm{M} \times \mathrm{N}$ variables $\mathrm{x}(\mathrm{i}, \mathrm{j})$ need to be computed from $\mathrm{M}+\mathrm{N}-1$ independent equations, which is an underdetermined problem.

Because of the fact that $\mathrm{M} \times \mathrm{N}-(\mathrm{M}+\mathrm{N}-1)$ equations are unknown, a large set of feasible solutions satisfy the equations (1), (2) and (3). This means that optimization techniques must be applied to find the most likely solution to the problem. We propose to utilize linear programming techniques [13], which can be adapted to our application.

We formulate the following optimization process:

$$
\text { Minimize F, with } F=\sum_{i=1}^{M} \sum_{j=1}^{N} c_{i j}^{(k)} \cdot x_{i j}^{(k)}
$$

under the constraints:

$$
\begin{array}{ll}
\sum_{j=1}^{N} x_{i j}^{(k)}=\alpha(i, k) & i=1, \ldots, M \\
\sum_{i=1}^{M} x_{i j}^{(k)}=\beta(j, k) & j=1, \ldots, N
\end{array}
$$

and

$$
1_{i j}^{(k)} \leqslant x_{i j}^{(k)} \leqslant u_{i j}^{(k)} \quad i=1, \ldots, M, j=1, \ldots, N(7)
$$

where $F$ represents the total cost for the reconstruction, $\mathrm{c}_{\mathrm{ij}}^{(\mathrm{k})}$ the cost coefficient associated with an increase in the grey value with one at position $(i, j)$ in 
slice $\mathrm{k}$. The parameters $\mathrm{u}_{\mathrm{ij}}^{(\mathrm{k})}$ and $\mathrm{l}_{\mathrm{ij}}^{(\mathrm{k})}$ are the upper and lower bounds for the grey value $\mathrm{x}_{\mathrm{ij}}^{(\mathrm{k})}$ at position $(i, j)$ in slice $k$; it is clear that $l_{i j}^{(k)} \geqslant 0$ and $u_{i j}^{(k)} \leqslant M I N$ $(\alpha(i, k), \beta(j, k))$ for all $i=1, \ldots, M, j=1, \ldots, N$, and for all $k$.

This optimization problem is related to the socalled Hitchcock or transportation problem, which can be approached conveniently as a flow problem in a directed network [13].

Fig. 8 illustrates the correspondence between the matrix reconstruction problem and the network flow problem. The capacities of the arcs directed away from the source are set equal to the row sums. The capacities of the arcs directed towards the sink are set equal to the column sums. The capacities of the intermediate arcs correspond with the entries of the matrix of the reconstruction problem. The flow through the network is maximal, if the actual flows through the source and sink arcs are equal to their capacities. For simplicity, only those intermediate arcs that do transport flow are given in Fig. 8 .

The a priori information can be brought into this network flow approach by assigning cost coefficients and capacities to the intermediate arcs. Intuitively, the assigment of the cost coefficients should follow the following pattern:

1. 'high' cost coefficients if low grey values are expected

2. 'medium' cost coefficients if medium grey values are expected

3. 'low' cost coefficients if high grey values are expected.

The reconstructed grey values in slice $\mathrm{k}-1$ can be used as a first approximation for the expected grey values and the related cost coefficients in the adjacent slice $k$. For the very first slice $k=1$, the cost coefficient model is defined by the data from the angiographic views; the grey values within the area defined by the endo- and epicardial boundaries are then evenly distributed. Similar rules may be applied to the capacities of the arcs in the network. The geometry of the cross section under reconstruction can also be incorporated in this network. For example, for those positions outside of the estimated epicardial boundary and inside of the
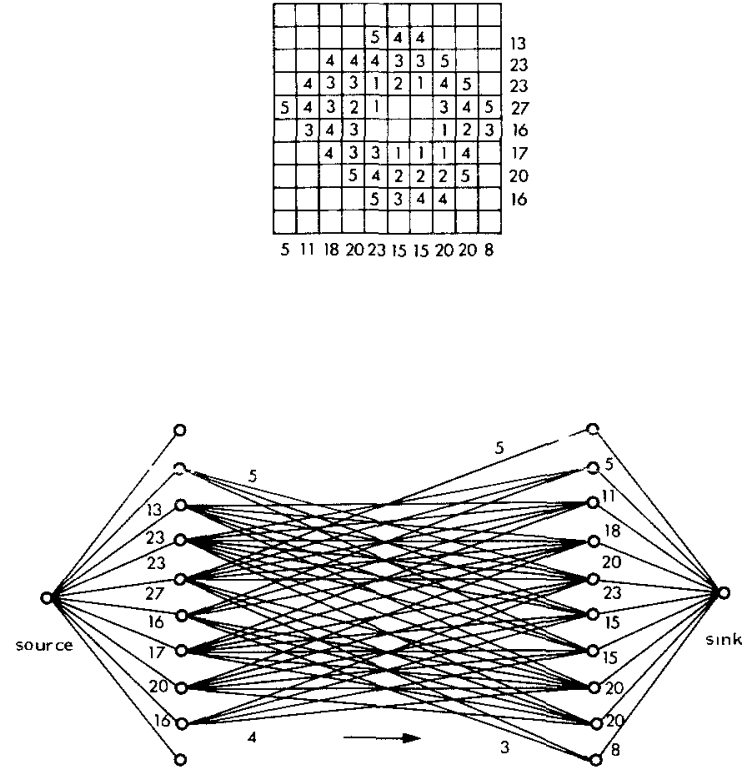

Fig. 8. The matrix reconstruction problem and the network flow approach.

estimated endocardial boundary, the lower and upper bounds of the capacities are set equal to zero. In this way the number of feasible solutions is significantly reduced, and thus the required processing time. For the other positions, the lower and upper bounds of the capacities are determined in correspondence to the cost coefficients.

Finally, algorithms from the field of Operation Research can be adapted to find the minimum cost solution at maximal flow through the capacitated network.

\section{First experimental results}

In the following paragraphs the first experimental results on the estimation of the 3D-geometry of the epicardial left ventricular boundary (paragraph 8.1) and the reconstruction of the myocardial perfusion (paragraph 8.2) are described.

\subsection{Estimation of the 3D-geometry of the epicardial boundary}

To determine which hull fitting technique provides 
the best results in the estimation of the epicardial boundary of a cross section based on the known intersection points with the coronary tree (paragraph 6.1.3), we used the known epicardial and ventricular luminal boundaries, and vessel intersection points of slices from postmortem hearts. Our material at the present time consists of two hearts, sliced perpendicular to the left ventricular long axis in a total of six slices of equal thickness. The epicardial and ventricular luminal boundaries of each slice were traced on paper and the intersection points of the visible vessels were indicated. The myocardial boundaries and the positions of the vessel intersection points were retraced from paper with a writing tablet and stored into the computer memory. To obtain a closed left ventricular myocardial boundary, the septal portion of the boundary was defined by the right ventricular cavity and by curves manually drawn between the right ventricular cavity and the nearby epicardial boundaries. From the hull fitting techniques that we have implemented (paragraph 6.1.3), the cubic B-spline was found to provide the best results. Fig. 9 shows the results for six slices of the first heart. This example shows that the computed hulls are acceptable in most slices, although not perfect. The mean difference and standard deviation of the differences expressed in mm's between the reconstructed and the true left ventricular myocardial boundaries for the slices of Fig. 5 are given in Table 1.

Two situations were investigated, one excluding
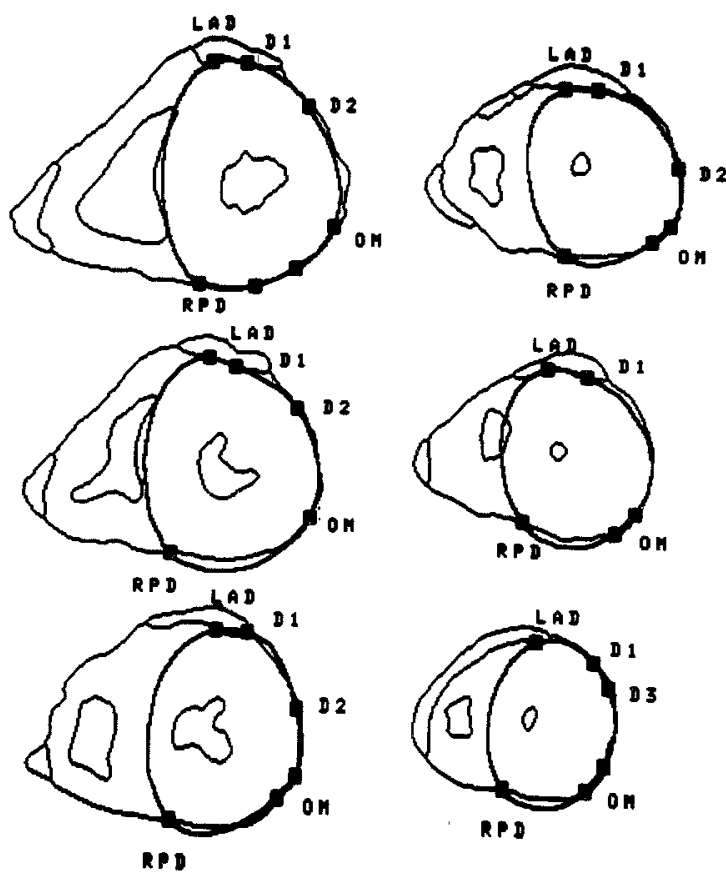

Fig. 9. Reconstructions of the outer boundaries of the left ventricular myocardium in six slices perpendicular to the left ventricular long axis on the basis of the intersection points of the slices with the coronary vessels by means of the cubic B-spline technique.

the position of the Right Posterior Descending branch in the reconstruction process, the other including this branch. For both cases the mean difference between the true and reconstructed boundaries were very small. However, for all cross sec-

Table 1. Statistical analysis of the differences between the computed (Fig. 9) and traced epicardial LV boundaries of the postmortem slices of Fig. 5.

\begin{tabular}{|c|c|c|c|c|c|}
\hline \multirow[b]{2}{*}{ Slice number } & \multirow[b]{2}{*}{$\begin{array}{l}\text { True circumference } \\
(\mathrm{mm})\end{array}$} & \multicolumn{2}{|c|}{$\begin{array}{l}\text { Excluding the right } \\
\text { posterior descending branch }\end{array}$} & \multicolumn{2}{|c|}{$\begin{array}{l}\text { Including the right } \\
\text { posterior descending branch }\end{array}$} \\
\hline & & $\begin{array}{l}\text { Mean difference } \\
(\mathrm{mm})\end{array}$ & $\begin{array}{l}\text { Standard deviation } \\
\text { of the } \\
\text { differences }(\mathrm{mm})\end{array}$ & $\begin{array}{l}\text { Mean difference } \\
(\mathrm{mm})\end{array}$ & $\begin{array}{l}\text { Standard deviation } \\
\text { of the } \\
\text { differences }(\mathrm{mm})\end{array}$ \\
\hline 1 & 236.6 & 0.4 & 3.1 & -0.6 & 0.9 \\
\hline 2 & 208.7 & 4.7 & 10.0 & -0.8 & 1.1 \\
\hline 3 & 207.6 & -0.5 & 2.5 & 0.2 & 2.3 \\
\hline 4 & 194.4 & -0.8 & 1.8 & 0.7 & 2.6 \\
\hline 5 & 168.8 & 1.4 & 2.0 & -0.1 & 0.9 \\
\hline 6 & 159.9 & -0.6 & 4.1 & 0.9 & 1.9 \\
\hline
\end{tabular}


tions, except for slice 4 , the standard deviations of the differences were clearly much smaller for the second case including the RPD-branch. Further refinements on the spline-technique and validations on larger series of experiments will have to be performed to find the generally acceptable solution for this problem.

\subsection{Reconstruction of myocardial perfusion}

To study the performance of the actual reconstruction process, an arbitrary model of myocardial perfusion was defined in a $64 \times 64$ matrix; from these data the corresponding biplane density profiles were computed. On the basis of these two density profiles, it was attempted to reconstruct the model. Both lower- and upperbound capacities were, rather arbitrarily, chosen symmetric to the model, such that clipping of values could not occur (section7). The cost coefficient model was defined from the density profiles by evenly distributing the values of the matrix. Before reconstruction a feasibility test was applied to the data, to ensure the existence of a non-empty solution space [13]. The linear programming algorithm has been implemented in the computer language TurboPascal and runs under the MS-DOS operating system. The optimal solution to the defined problem was found in about 250 s on a COMPAQ AT personal computer (clock frequency $12 \mathrm{MHz}$ ).

Although both the cost coefficient model and the lower- and upperbound capacities were chosen rather arbitrarily, we believe that an acceptable reconstruction can be obtained by choosing these values properly. How this must be done is still a matter of research.

\section{Conclusions}

In this paper we have proposed our approach for the $3 \mathrm{D}$ reconstruction of the myocardial perfusion from biplane angiograms. The following five major steps can be distinguished: 1) determination of the optimal biplane angiographic views; 2) myocardial perfusion image acquisition; 3) preprocessing of the selected myocardial perfusion images; 4) estimation of the 3D-geometry of the endo- and epicardial boundaries of the left ventricular myocardium; and 5) reconstruction of the regional myocardial perfusion in the different cross sections perpendicular to the left ventricular long axis. From our present experiences we conclude the following:

1. 3D-reconstruction of the myocardial perfusion from biplane angiographic views is a strongly underdetermined problem. Therefore, a priori knowledge of the myocardium must be utilized in the reconstruction process.

2. It is proposed to use the geometry of the endoand epicardial boundaries of the myocardial cross sections as part of the a priori knowledge.

3. From the different hull-fitting techniques implemented, the cubic B-spline fitting technique showed the best results in the estimation of the epicardial boundaries of the cross sections from the arterial intersection points.

4. Reconstruction of the myocardial perfusion by means of linear programming techniques shows great promise, provided the proper cost coeficient model and lower- and upperbound capacities are defined.

\section{Acknowledgements}

The authors wish to thank Dr. C. Roos, Department of Mathematics and Informatics, Delft University of Technology for his contributions to the 3D-reconstruction problem and Mrs. F. van den Ende for her secretarial contribution in the preparation of this manuscript. This project is supported by the Dutch Heart Foundation under grant no. NHS 86.081.

\section{References}

1. Reiber JHC. Morphologic and densitometric analysis of coronary arteries. In: Heintzen $\mathrm{PH}$, ed. Progress in digital angiocardiography. Boston/Dordrecht: Martinus Nijhoff Publishers, 1987 (in press).

2. Gould KL, Kirkeeide RL. Assessment of stenosis severity. In: Reiber JHC, Serruys PW, eds. State of the art in quanti- 
tative coronary arteriography. Dordrecht/Boston/Lancaster: Martinus Nijhoff Publishers, 1986: 209-228.

3. Zijlstra F, Ommeren J van, Reiber JHC, Serruys PW. Does quantitative assessment of coronary artery dimensions predict the physiological significance of a coronary stenosis? Circ 1987; 75: 1154-1161.

4. Vogel R, LeFree M, Bates E, O'Neill W, Foster R, Kirkin P, Smith D, Pitt B. Applications of digital techniques to selective coronary arteriography: use of myocardial contrast appearance time to measure coronary flow reserve. Am Heart J 1984; 107: 153-164.

5. Wilson RF, White CW. Intracoronary papaverine: an ideal coronary vasodilator for studies of the coronary circulation in conscious humans. Circ 1986; 73: 444-451.

6. Zijlstra F, Serruys PW, Hugenholtz PG. Papaverine: the ideal coronary vasodilator for investigating CFR? A study of timing magnitude, reproducibility and safety of the coronary hyperemic response after intracoronary papaverine. Cath and Cardiovasc Diagn 1986; 12: 298-303.

7. Ommeren J van, Zijlstra F, Serruys PW, Reiber JHC. A rapid angiographic technique to measure relative coronary blood flow. In: Young IT, Biemond J, Duin RPW, Gerbrands JJ, eds. Signal processing III: Theories and applications. Amsterdam/New York/Oxford/Tokyo: North-Holland, 1986: 1375-1378.

8. Dumay ACM. 3D-reconstruction from two orthogonal projections with linear programming techniques. Thesis, Information Theory Group. Delft University of Technology, June 1986 (in Dutch).

9. Onnasch DGW. Reconstruction of the spatial distribution of myocardial perfusion from multiple-view arteriography. In: Heintzen P, ed. Progress in digital angiocardiography. Boston/Dordrecht: Martinus Nijhoff Publishers, 1987 (in press).

10. Slump CH, Gerbrands JJ. A network flow approach to the reconstruction of the left ventricle from two projections. Comp Graph and Im Proc 1982; 18: 18-36.

11. Reiber JHC, Gerbrands JJ, Troost GJ, Kooijman CJ, Slump CH. 3D-reconstruction of coronary arterial segments from two projections. In: Heintzen PH, Brennecke $\mathrm{R}$, eds. Digital imaging in cardiovascular radiology. Stuttgart: Georg Thieme Verlag, 1983: 151-163.

12. Gerbrands JJ, Slump CH. 3D-reconstruction of homogeneous objects from two Poisson-distributed projections. Patt Recogn Letters 1985; 3: 137-145.

13. Wagner HM. Principles of operations research, with applications to managerial decisions. 2nd ed. London: Prentice/ Hall Int, 1975.
14. Reiber JHC, Serruys PW, Slager CJ. Quantitative coronary and left ventricular cineangiography; methodology and clinical applications. Boston/Dordrecht/Lancaster: Martinus Nijhoff Publishers, 1986.

15. Reiber JHC, Zijlstra F, Ommeren J van, Serruys PW. Relation between coronary flow reserve and severity of coronary obstruction, both assessed from coronary cineangiogram. In: Heintzen PH, ed. Progress in digital angiocardiography. Dordrecht: Martinus Nijhoff Publishers, 1987 (in press).

16. Gerbrands JJ, Reiber JHC, Kooijman CJ, Rademaker RT. Model-guided segmentation of coronary cine-angiograms. In: Schüssler HW, ed. Signal Processing II: Theories and applications. Amsterdam/New York/Oxford/Tokyo: North-Holland, 1983: 601-604.

17. Langhout G. Structurele analyse van de kransslagaderboom uit röntgenfilms. Thesis, Information Theory Group, Faculty of Electrical Engineering, Delft University of Technology, May 1982 (in Dutch).

18. Minderhoud $\mathbf{H}$. Onderzoek naar en implementatie van omhullende-techniẹken ten behoeve van de reconstructie van het spierweefsel van de linker-hartkamer. Thesis, Information Theory Group, Faculty of Electrical Engineering, Delft University of Technology, June 1987 (in Dutch).

19. Leeuwen PJ van, Reiber JHC. Automated detection of left ventricular boundaries from $35 \mathrm{~mm}$ contrast cineangiograms. In: Young IT, Biemond J, Duin RPW, Gerbrands JJ, eds. Signal processing III: Theories and applications. Amsterdam/New York/Oxford/Tokyo: North-Holland, 1986: 1409-1412.

20. Eiho S, Kuwahara M. 3D-reconstruction of the left ventricle. Preceedings Eight Annual Conference of the IEEE. Engineering and Biology Society, IEEE Cat no. 86CH2368-9, 1986: 112-115.

21. Onnasch DGW, Schmitz W, Heintzen PH. Problems of the binary reconstruction of the left ventricle from biplane angiocardiograms. In: Heintzen PH, Brennecke R, eds. Digital imaging in cardiovascular radiography. Stuttgart, New York: Georg Thieme Verlag, 1983: 141-151.

Address for offprints:

Johan H.C. Reiber, Ph.D.

Erasmus University

C.V.R. EE 2328

P.O. Box 1738

3000 DR Rotterdam

The Netherlands 